\title{
CARACTERIZACIÓN PRODUCTIVA DE LA GALLINA DE SOBRARBE
}

\author{
PRODUCTIVE CHARACTERIZATION OF SOBRARBE HEN
}

\author{
Cajal, J.R. ${ }^{1 *}$ y Francesch, A. ${ }^{2}$
}

${ }^{1}$ Asociación de Criadores de Gallina del Sobrarbe (AGASOB). Sariñena, Huesca. España. jrcajal@yahoo.es ${ }^{2}$ Instituto de Investigación y Tecnología Agroalimentaria (IRTA). Constantí, Tarragona. España.

\section{PALABRAS CLAVE ADICIONALES}

Aves de corral. Crecimiento. Producción de huevos. Razas autóctonas. Rendimiento de canal.

\section{RESUMEN}

Se aborda la caracterización productiva comparativa de las variedades negra y trigueña de la gallina de Sobrarbe, en lo referente a crecimiento (ganancia de peso, índices de conversión y rendimientos de canal), y puesta (número y calidad de los huevos). Los pollos machos alcanzaron los 2,1 $\mathrm{kg}$ de peso a las 19 semanas, con un índice de conversión de 4,5 y rendimiento de canal eviscerada cercano al 83 \%. La puesta comenzo a las 20 semanas, con un resultado anual de 170 huevos. Solo se observaron diferencias significativas entre variedades, en las Unidades Haugh.

\section{SUMMARY}

In this work is done an approach to the productive characterization of the breed Sobrarbe hen, so much in the growth (study of the growth, indexes of conversion and carcass) as in the lay phase (number and quality of eggs). The males reached $2,1 \mathrm{~kg}$ of live weight at 19 weeks of life, with an index of conversion of 4,5 and a performance of the eviscerated carcass close to $83 \%$. Hens started laying at 20 weeks of age, with an annual result of 170 eggs. Significant differences between varieties were only observed in the Haugh Units.

\section{INTRODUCCIÓN}

La actual raza de gallinas del Sobrarbe tiene una historia relativamente corta. En

Financiado con una beca del Instituto de Estudios Altoaragoneses. Diputación Provincial de Huesca.

\section{Additional KEYWORDS}

Carcass yield. Egg production. Growth. Poultry. Native breeds.

1995 comenzó el trabajo de recopilación de un grupo racial hallado en el norte de Huesca (Azón y Francesch, 1997), que resultó en una población base con diferentes colores de plumaje en la que inicialmente se definieron seis variedades de color según el estándar racial (Francesch, 2006).

Los objetivos del presente trabajo son conocer la capacidad productiva de la raza de gallinas del Sobrarbe en el crecimiento y rendimiento a la canal de los pollos, y en la puesta (edad a la entrada en producción, número y calidad comercial de huevos) comparando las variedades negra y trigueña y, en la medida de lo posible, con otras razas de gallinas españolas en las que se han realizado estudios similares.

\section{MATERIAL Y MÉTODOS}

Se trabajó con ejemplares de las variedades trigueña y negra con manto pajizo. Los pollitos al nacer fueron sexados e identificados con una banda numerada insertada en la membrana alar. Posteriormente se separaron en cuatro lotes: 62 pollitas trigueñas, 61 pollos trigueños, 46 pollitas negras y 48 pollos negros. El trabajo se desarrolló en dos fases: crecimiento y puesta.

Dietas. A los pollos se les suministró un pienso del 22,99\% de proteína con $3010 \mathrm{kca} /$ kg EM hasta los 7 días de vida; otro del 21,37 
$\%$ de proteína con $3060 \mathrm{kcal} / \mathrm{kg}$ EM de los 7 a los 28 días y finalmente otro del 19,22\% de proteína con $2930 \mathrm{kcal} / \mathrm{kg}$ hasta sacrificio.

A las pollitas se les suministró un pienso del $22,99 \%$ de proteína con $3010 \mathrm{kca} / \mathrm{kg}$ EM hasta los 7 días de vida; otro del 15,92\% de proteína con $2800 \mathrm{kcal} / \mathrm{kg}$ EM de los 7 a los 28 días y f i n a 1 m e n t e otro del 15,90\% de proteína con $2775 \mathrm{kcal} / \mathrm{kg}$ durante todo el período de puesta.

Crecimiento. Los pollos se pesaron individualmente desde el día siguiente al nacimiento con una periodicidad semanal, hasta el sacrificio en pollos y la entrada en puesta de las pollitas. Para el estudio de canales se sacrificaron 12 pollos por lote a las 19 semanas de vida, siguiendo las directrices de disección de canales que marca la WPSA (World's Poultry Science Association, 1984).

La alimentación fue ad libitum, registrando el consumo de alimento de cada lote en el momento de realizar las pesadas de los animales.

Puesta y calidad del huevo. Una vez detectada la primera puesta, se hicieron dos controles diarios de puesta y registro de estos datos, hasta las 72 semanas de vida de las gallinas.

Tabla I. Consumo acumulado $(C A, g)$, peso vivo $(g) e$ índice de conversión acumulado (IC) en dos poblaciones de pollos (negra y trigueña) del Sobrarbe. (Cumulative consumption (CA, g), live weight $(\mathrm{g})$ and cumulative conversion index (IC) in two populations of male chickens of Sobrarbe hens from the varieties black and weathen).

\begin{tabular}{ccccccc}
\hline & \multicolumn{3}{c}{ Pollos negros } & \multicolumn{3}{c}{ Pollos trigueños } \\
Edad & CA & Peso & IC & CA & Peso & IC \\
\hline 3 & 411,6 & 256,4 & 1,9 & 377,0 & 239,8 & 1,9 \\
8 & 2532,1 & 876,9 & 3,0 & 2406,7 & 849,7 & 3,0 \\
11 & 4383,5 & 1310,5 & 3,4 & 4162,6 & 1272,7 & 3,4 \\
19 & 9771,4 & 2116,7 & 4,6 & 9040,8 & 2106,0 & 4,4
\end{tabular}

${ }^{1}$ semanas.
Tabla II. Consumo acumulado (CA, g), peso vivo $(g)$ en dos poblaciones (negra y trigue$\tilde{n} a$ ) de pollitas del Sobrarbe. (Cumulative feed consumption (CA, g) and live weight (g), in two populations of female chickens of black and weathen Sobrarbe hens).

\begin{tabular}{ccccc}
\hline \multicolumn{4}{c}{ Pollitas negras } & \multicolumn{3}{c}{ Pollitas trigueñas } \\
Edad semanas & CA & Peso & CA & Peso \\
\hline 3 & 416,9 & 221,1 & 384,0 & 223,6 \\
8 & 2083,3 & 534,3 & 1680,1 & 559,2 \\
11 & 3726,8 & 800,0 & 3341,2 & 825,7 \\
20 & 7871,7 & 1487,0 & 7596,5 & 1482,0 \\
52 & 27983,5 & 1752,6 & 27194,6 & 1740,9 \\
\hline
\end{tabular}

El estudio de la calidad del huevo se hizo con los huevos puestos por cada lote el día en que las gallinas cumplían las 25, 30, 52 y 72 semanas de vida. Consistía en registrar el peso de los huevos, el peso y espesor de la cáscara, el color de la cáscara con un reflectómetro y de la yema (por comparación con la escala Roche), así como la altura del albumen para calcular las unidades Haugh (calidad del albumen).

Análisis estadístico. En el tratamiento estadístico de los datos de peso de los animales, se aplicó un modelo con la variedad y el sexo como factores, con su interacción. En el tratamiento de los datos del estudio de canales, se utilizó un modelo con la variedad como factor. Los porcenta-

Tabla III. LS means de los rendimientos de canal, en pollos (negros, $N$ y trigueños $T$ ) de la raza Sobrarbe. (Ls means of the carcass yield, in males of the varieties black $(\mathrm{N})$ and weathen ( $T$ ) of chickens of the Sobrarbe breed).

\begin{tabular}{lcc}
\hline & $\mathrm{N}(\mathrm{n}=12)$ & $\mathrm{T}(\mathrm{n}=12)$ \\
\hline Matadero & $90,50 \pm 0,99$ & $92,11 \pm 0,92$ \\
Canal eviscerada & $81,80 \pm 0,94$ & $84,29 \pm 0,80$ \\
Muslos & $13,48 \pm 0,12$ & $13,85 \pm 0,12$ \\
Contramuslos & $15,53 \pm 0,22$ & $15,17 \pm 0,27$ \\
Alas & $10,74 \pm 0,09$ & $10,00 \pm 0,18$ \\
Pechugas & $12,71 \pm 0,17$ & $13,53 \pm 0,25$ \\
\hline
\end{tabular}


jes fueron analizados previa transformación al arco seno de la raíz cuadrada de su tanto por uno.

En el tratamiento estadístico de los datos de calidad del huevo, se consideraron como factores la variedad y la edad, junto con la interacción entre ambos.

Para resolver éstos modelos se utilizó un modelo lineal general (GLM) del paquete estadístico SAS versión 9.1.

\section{RESULTADOSYDISCUSIÓN}

Pesos, consumos e índice de conversión. En las tablas I y II, se muestran los resultados de peso y consumo durante el desarrollo de los pollos y las pollitas en fechas clave del crecimiento. Como era previsible, no se observaron diferencias significativas entre los pesos de las variedades y sí entre sexos. Los pollos alcanzaron los $2,1 \mathrm{~kg}$ (mínimo peso comercial) a las 19 semanas de vida. Este dato de crecimiento, permite calificar a los ejemplares de la raza de gallinas Sobrarbe de crecimiento lento, al igual que la raza Castellana (Miguel, 2003) y los tipos tradicionales de las razas Penedesenca negra, Empordanesa roja y Prat leonada (Escoda, 2004). En las pollitas se observa un enlentecimiento del crecimiento a partir de las 20 semanas quedando con un peso adulto (a las 52 semanas) en torno a 1,7 kg. Este peso, que corresponde al de una raza ligera, es inferior al observado en otras razas españolas que situadas en su grupo como las catalanas y la Menorca, en las que las gallinas adultas pueden sobrepasar los $2 \mathrm{~kg}$ (Francesch, 2006 y Villalba et al., 2007).

Tampoco se observaron diferencias significativas entre variedades en los índices de conversión y consumo. El índice de conversión hasta alcanzar los $2,1 \mathrm{~kg}$ de peso vivo se ha mantenido por debajo de 5 en la raza Sobrarbe. De acuerdo con los datos disponibles en la bibliografía, este es el mejor índice de conversión dentro del grupo de razas en las que, por su peso, se encuadraría la Sobrarbe.

Rendimiento de la canal de los pollos. En la tabla III se presentan los rendimientos de canal y de distintas partes de las canales de los pollos. No se observaron diferencias estadísticamente significativas entre variedades en rendimientos de las distintas partes de la canal. Los resultados obtenidos en este apartado son similares a los de las razas catalanas (Escoda, 2004) y la raza Castellana (Miguel, 2003).

Puesta. Esta fase duró de las 20 a las 72 semanas de vida. En la tabla IV se aportan los datos. La puesta media se situó entorno a los 170 huevos por gallina y año, con un índice de conversión/docena similar al de las razas catalanas y considerablemente mejor al 5,1 obtenido en la raza Menorca (Villalba et al., 2005). La puesta observada en la raza Sobrarbe fue cercana a la de 180 huevos obtenida en la raza Euskal Oiloa (Francesch y Atxa, 2004), a la estimada en 163 huevos en la raza Castellana (Miguel, 2003) y a la de 170 huevos de la raza Prat (Francesch et al., 1998). Se han observado puestas inferiores, entorno a los 120 huevos, en la raza Menorca (Villalba et al., 2007) y la Mos (Sánchez et al., 2000). Puestas superiores a la de la raza Sobrarbe se han alcanzado en la raza Extremeña, con 200 huevos (Muriel, 2002), así como en la Penedesenca negra con 200 huevos y en la Empordanesa roja con 220 (Francesch et al., 1998).

Calidad del huevo. Los datos que se muestran en la tabla $\mathbf{V}$ son las medias de

Tabla IV. Resultados durante la fase de puesta (0-72 semanas) en las variedades Negra, Ny Trigueña, T de gallinas Sobrarbe. (Results during the laying period in the varieties black (N) and weathen ( $\mathrm{T}$ ) of Sobrarbe hens).

\begin{tabular}{lcc}
\hline & $\mathrm{N}$ & $\mathrm{T}$ \\
\hline Huevos acumulados & 167 & 173 \\
Consumo (kg/gallina) & 45,79 & 45,53 \\
Conversión (kg pienso/docena) & 3,29 & 3,15 \\
\hline
\end{tabular}


Tabla $\boldsymbol{V}$. LS means de los parámetros de calidad del huevo en las variedades negra (N) y trigueña (T) de Gallinas Sobrarbe. (Ls means of the egg quality parameters in the varieties black $(\mathrm{N})$ and weathen $(\mathrm{T})$ of the Sobrarbe hens).

\begin{tabular}{lcc}
\hline & $\mathrm{N}$ & $\mathrm{T}$ \\
\hline Peso huevo g & $51,90 \pm 0,87$ & $50,45 \pm 0,76$ \\
Peso cáscara g & $4,52 \pm 0,11$ & $4,68 \pm 0,09$ \\
Espesor cáscara mm & $0,34 \pm 0,007$ & $0,36 \pm 0,006$ \\
Unidades Haugh & $89,23 \pm 1,27^{\mathrm{a}}$ & $85,49 \pm 1,11^{\mathrm{b}}$ \\
Color yema Roche & $11,91 \pm 0,28$ & $12,03 \pm 0,25$ \\
Color cáscara & $41,27 \pm 1,77$ & $44,89 \pm 1,55$ \\
\hline
\end{tabular}

todos los huevos evaluados durante la fase de puesta. Excepto para el peso del huevo y el color de la cáscara, no hay referencias bibliográficas en razas de gallinas españolas para los demás parámetros. Se observaron, sorprendentemente, diferencias significativas, entre variedades, respecto a las Unidades Haugh, aunque en ambos casos los valores fueron altos variando la calidad entre muy buena y excelente El peso de los huevos es relativamente bajo y permitiría su

\section{BIBLIOGRAFÍA}

Azon, R. y Francesch, A. 1997. Recuperación y definición de poblaciones de gallinas del pirineo altoaragones. Comunicaciones de las II Jornadas de Etnologia Avícola (II). Arte Avícola, 19: 12-14.

Escoda, L. 2004. Estudio comparado de características productivas y de calidad de la canal y de la carne en pollos obtenidos de razas autóctonas catalanas. Tesis Doctoral. Universidad de Barcelona. España.

Francesch, A.; Casanovas, P. and Fontgibell, A. 1998. Heterosis in Catalan poultry genetic stocks under egg production traits selection. Proc. of the $6^{\text {th }}$ World Congress on Genetics applied to Livestock Production. Kusadassi. Turquía. Vol. 24: 298-301.

Francesch, A. y Atxa, E. 2004. Posibilidades de la raza de gallinas Euskal Oiloa para responder a la selección por puesta. IV Congreso Ibérico sobre Recursos Genéticos Animales. Ponte de comercialización en la categoría de medianos (M), al igual que ocurre en otras razas de gallinas españolas antes mencionadas. Como razas que ponen un huevo de mayor tamaño podemos citar la Menorca (Villalba et al., 2007) y la Prat (Francesch et al., 1998), en las que a partir de las 40 semanas de vida de la gallina, el peso medio del huevo supera $\operatorname{los} 60 \mathrm{~g}$.

El color de la cáscara del huevo, en la Sobrarbe, es similar al de la raza Prat (Francesch et al., 1998) y mucho más claro, del orden de 30 unidades menos de $\%$ de absorción de la luz, que en la Penedesenca y la Empordanesa (Francesch et al., 1998). Un color muchos más claro, con cáscara casi blanca se obtiene en la Castellana y en la Menorca.

\section{CONCLUSIONES}

La raza de gallinas Sobrarbe engloba a animales ligeros de crecimiento lento y precocidad sexual alta. Las variedades negra y trigueña de la raza son similares en crecimiento y puesta.

Lima. Portugal.

Jensen, J.F. 1984. Method of dissection of broiler carcases and description of parts. World's Poultry Science Association European Federation. Working Group V.

Miguel, J.A. 2003. Caracterización productiva y genética de una población de gallinas de raza Castellana Negra. Tesis doctoral. Universidad de Valladolid. España.

Muriel, A. 2002. Estudio de diversos parámetros productivos en la raza de gallinas Extremeña azul. Arte Avícola, 48: 17-19.

Sánchez, L.; Sánchez, B. y Fernández, B. 2000. Programa de preservación de la gallina de raza Mos en Galicia. Arch Zootec, 49: 77-80.

Villalba, D.; Francesch, A; Pons, A; Bustamante, J.; Espadas, M.; Santonja, V. y Cubiló, D. 2007. Resultados de puesta y crecimiento en una población de gallinas de raza Menorca. Arch Zootec, 56 (Supl.) 1: 373-804.

Archivos de zootecnia vol. 63, núm. 241, p. 214. 